\title{
SYNAPTIC RELEASE OF EXCITATORY AMINO ACID NEUROTRANSMITTER MEDIATES ANOXIC NEURONAL DEATH ${ }^{1}$
}

\author{
STEVEN ROTHMAN \\ Departments of Pediatrics, Neurology, and Anatomy and Neurobiology, Washington University School of Medicine, \\ St. Louis, Missouri 63110
}

Received December 27, 1983; Revised February 16, 1984; Accepted February 27, 1984

\begin{abstract}
The pathophysiology of hypoxic neuronal death, which is difficult to study in vivo, was further defined in vitro by placing dispersed cultures of rat hippocampal neurons into an anoxic atmosphere. Previous experiments had demonstrated that the addition of high concentrations of magnesium, which blocks transmitter release, protected anoxic neurons. These more recent experiments have shown that $\gamma$-D-glutamylglycine (DGG), a postsynaptic blocker of excitatory amino acids, was highly effective in preventing anoxic neuronal death. DGG also completely protected the cultured neurons from the toxicity of exogenous glutamate (GLU) and aspartate (ASP). In parallel physiology experiments, DGG blocked the depolarization produced by GLU and ASP, and dramatically reduced EPSPs in synaptically coupled pairs of neurons. These results provide convincing evidence that the synaptic release of excitatory transmitter, most likely GLU or ASP, mediates the death of anoxic neurons. This result has far-reaching implications regarding the interpretation of the existing literature on cerebral hypoxia. Furthermore, it suggests new strategies that may be effective in preventing the devastating insults produced by cerebral hypoxia and ischemia in man.
\end{abstract}

Cerebral hypoxia, either as an isolated event, or as a concomitant of occlusive cerebrovascular disease, perinatal asphyxia, or cardiorespiratory failure, is a frequent cause of human neurological injury. On occasion, the deficits produced by hypoxia are reversible, but irreversible brain damage is a far more common event. Despite the importance of the problem, the pathophysiology of hypoxic brain injury has not yet been determined, although it appears that altered calcium homeostasis, elevated free fatty acid concentrations, accumulation of eicosanoids, and increased extracellular lactic acid all contribute to neuronal death (Siesjo, 1981; Raichle, 1983). However, resolving all of the factors responsible for pure hypoxic brain injury has remained difficult because hypoxia in vivo is invariably associated with hypotension, hypercarbia, and acidosis, which are all potentially damaging.

Recent in vitro observations have provided new insights into the pathophysiology of neuronal injury pro-

\footnotetext{
${ }^{1}$ This work was supported by National Institute of Neurological and Communicative Disorders and Stroke Grants NS00568 and NS19988 and by the Epilepsy Foundation of America. I would like to thank Drs. Gerald Fischbach, Richard Hume, Marcus Raichle, and Joseph Volpe, who critiqued earlier drafts of this manuscript; Irene Karl, Ph.D., who provided the glutamate assay; Miriam Samaie, who provided excellent technical help; Robert Freund, who did the photography; and Kim Kendall, who typed the paper.
}

duced by hypoxia, unaccompanied by other complicating variables. Experiments with fetal rat hippocampal neurons maintained in dispersed tissue culture showed that synaptic activity mediated neuronal death when the cultures were exposed to an anoxic environment (Rothman, 1983). Freshly plated neurons, which had not yet formed synapses, were very resistant to prolonged anoxia. Mature cultures markedly deteriorated in the absence of oxygen, and within a few hours all of the neurons had been replaced by debris. However, when synaptic activity was blocked by high concentrations of magnesium, the cultures tolerated prolonged anoxia and showed virtually no morphological changes.

While these experiments clearly demonstrated that synaptic activity was responsible for killing hypoxic neurons, they failed to indicate the mechanism of activityinduced neuronal death. The experiments described in the present paper provide convincing evidence that synaptically released neurotransmitter, most likely glutamate (GLU) or aspartate (ASP), initiates the cascade of reactions that culminates in neuronal death in hypoxia.

\section{Materials and Methods}

Culture. Hippocampi were dissected from the brains of 18-day-old rat fetuses under sterile conditions and were placed in $0.1 \%$ trypsin (Gibco) in calcium-magnesiumfree Hanks' Balanced Salt Solution (HBSS) buffered to 
pH 7.3 with $10 \mathrm{~mm}$ HEPES. After a 15 -min incubation at $37^{\circ} \mathrm{C}$, the hippocampi were washed three times with HBSS, resuspended in approximately $1 \mathrm{ml}$ of HBSS, and dissociated by passage through a series of Pasteur pipettes with flame-narrowed tips. The cells were then pipetted into $35-\mathrm{mm}$ tissue culture dishes (Falcon) at a density of approximately 1.5 to $2.0 \times 10^{4}$ cells $/ \mathrm{cm}^{2}$. Each dish contained $1.5 \mathrm{ml}$ of medium.

The dishes were precoated with poly-L-lysine hydrobromide, molecular weight 3.0 to $7.0 \times 10^{4}$ (Sigma), as previously described (Banker and Cowan, 1977) except that a solution of only $0.01 \%$ poly-L-lysine in borate buffer was used.

The culture medium contained equal volumes of Ham's F-12 and Dulbecco's Modified Eagle's Medium supplemented with an additional $120 \mathrm{mg} / 100 \mathrm{ml}$ of glucose, 5 $\mu \mathrm{g} / \mathrm{ml}$ of bovine insulin, $100 \mu \mathrm{g} / \mathrm{ml}$ of human transferrin, $20 \mathrm{nM}$ progesterone, $100 \mu \mathrm{M}$ putrescine, and $30 \mathrm{nM}$ selenium dioxide (all from Sigma). Penicillin and streptomycin (both from Gibco) were added to final concentrations of $20 \mathrm{units} / \mathrm{ml}$ and $20 \mu \mathrm{g} / \mathrm{ml}$, respectively. The final medium also contained $10 \%$ heat-inactivated human serum. All recent experiments have employed my own serum, to eliminate the variability seen with different lots of commercial animal sera. However, fetal calf and horse sera have supported adequate neuronal growth and survival in the past. This medium is almost identical to the previously described N2 (Bottenstein and Sato, 1979), except that it omits HEPES and contains serum.

Cultures were maintained in a humidified incubator with $5 \% \mathrm{CO}_{2}$ at $36^{\circ} \mathrm{C}$. After 4 to 6 days, division of nonneuronal cells was halted by the addition of $15 \mu \mathrm{g} / \mathrm{ml}$ of fluorodeoxyuridine and $35 \mu \mathrm{g} / \mathrm{ml}$ of uridine (Ransom et al., 1977). the growth medium was never changed, and cultures were not refed. Cultures maintained in this manner usually survived 4 to 5 weeks, and this was not demonstrably affected by refeeding.

Induction of anoxia. In early experiments, cultures were made anoxic by placing them in a home-made chamber which was flushed with $95 \% \mathrm{~N}_{2} / 5 \% \mathrm{CO}_{2}$, sealed, and returned to the incubator. More recently, a commercially available incubator chamber specifically designed for this purpose has been employed (Billups-Rothenberg, Del Mar, CA). Dishes filled with water were placed into both chambers to maintain humidity as close to $100 \%$ as possible.

Individual microscope fields were photographed prior to anoxia and then relocated for photography after varying periods of anoxia. Just prior to anoxic exposure, medium was replaced with Earle's Minimum Essential Medium (MEM) or MEM supplemented with $10^{-2} \mathrm{M} \gamma$ D-glutamylglycine (DGG; Cambridge Research Biochemicals LTD, Atlantic Beach, NY) as the sodium salt.

Excitatory amino acid toxicity. In these experiments, individual microscope fields were photographed. The medium was then replaced with MEM, to which had been added $10^{-4} \mathrm{M}$ ASP or $10^{-4} \mathrm{M}$ GLU, either alone or in combination with $10^{-2}$ M DGG. The same fields were then relocated for photography at set intervals.

Physiology. For intracellular recording, culture medium was replaced with HBSS modified to contain $3 \mathrm{mM}$ potassium, $5 \mathrm{mM}$ calcium, $1 \mathrm{mM}$ magnesium, and $10 \mathrm{mM}$
HEPES ( $\mathrm{pH}$ 7.3). Tetrodotoxin (TTX), $1 \mu \mathrm{g} / \mathrm{ml}$, was added to block action potentials in some experiments. The culture was transferred to the stage of an inverted, phase contrast microscope, and the temperature was maintained at $35^{\circ} \mathrm{C}$ by a heater insert connected to a feedback system. Evaporation was prevented by covering the surface of the dish with a layer of surgical mineral oil, which allows for adequate gas exchange. Neurons were impaled under direct vision at $\times 500$ magnification. Microelectrodes were filled with $4 \mathrm{M}$ potassium acetate and had impedances between 40 and 80 megohms. A standard bridge circuit was used for recording and current passage.

GLU, ASP, and DGG were delivered by microperfusion (Choi and Fischbach, 1981). They were dissolved in medium identical to the bath and applied by pressure from blunt-tipped pipettes $(\sim 5 \mu \mathrm{m})$ located approximately $30 \mu \mathrm{m}$ from impaled neurons. A pulse generator activating a solenoid valve (General Valve Corp., Fairfield, $\mathrm{NJ}$ ) controlled ejection duration.

\section{Results}

In five control cultures exposed to $95 \% \mathrm{~N}_{2} / 5 \% \mathrm{CO}_{2}$, the neurons had markedly degenerated by 6 to $8 \mathrm{hr}$ (Fig. $1, A 1$ and A2). After 1 day it was impossible to delineate outlines of neurons except for their processes, which appeared swollen and vacuolated (Fig. 1, A3). Although the figures show only a single microscope field followed over time, they are representative of the appearance of all five control cultures; no intact neurons were seen after 6 to $8 \mathrm{hr}$. There was a delay of a few hours before the effects of anoxia became obvious. This is most likely because some oxygen remained dissolved in the medium. When aerobic metabolism was blocked by cyanide rather than anoxia, the effects were manifest within $1 \mathrm{hr}$ (Rothman, 1983).

In contrast, glia, which are also present in these hippocampal cultures (Rothman and Cowan, 1981; Seifert et al., 1981), appear relatively resistant to anoxia, at least over the period of observation. They are not easy to distinguish in the figures because they tend to grow as a monolayer beneath the neurons.

As previous experiments had indicated that blockade of transmitter release by magnesium prevented neuronal death in these cultures, it seemed possible that released transmitter might be responsible for this phenomenon (Rothman, 1983). The most likely excitatory transmitters used by hippocampal neurons in these cultures are GLU and ASP (Rothman, 1982), which are well known neurotoxins (Olney, 1978).

To test the hypothesis that released GLU or ASP produced the neuronal loss, five cultures were treated with $10^{-2}$ M DGG, a postsynaptic blocker of excitatory amino acids (Francis et al., 1980), and were then exposed to $95 \% \mathrm{~N}_{2} / 5 \% \quad \mathrm{CO}_{2}$. There were dramatic differences between all five DGG- treated cultures and control cultures viewed simultaneously. In the former, almost all neurons appeared healthy after $8 \mathrm{hr}$ and 1 day (Fig. 1, $B 1, B 2$, and $B 3$ ), although the cell bodies of a few neurons were no longer evident. The contrast between DGGtreated cultures and controls was so evident that it was not further quantitated. Similar experiments attempted 

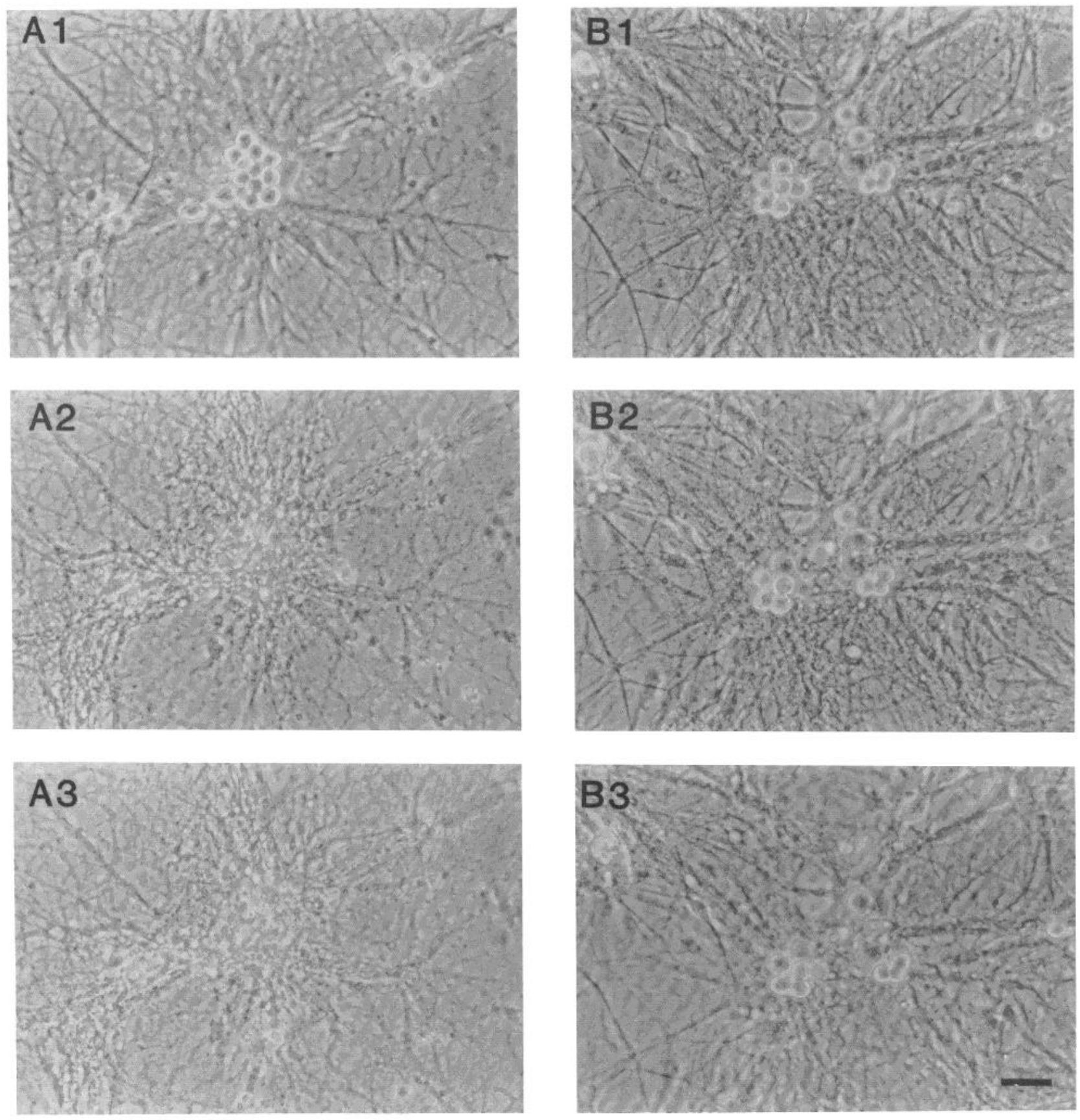

Figure 1. Effects of anoxia on cultured hippocampal neurons. Cultures were removed from the standard $5 \% \mathrm{CO}_{2}$ atmosphere, and medium was replaced with MEM. They were then placed into a container which was flushed with $95 \% \mathrm{~N}_{2} / 5 \% \mathrm{CO}_{2}$ and resealed. Representative fields of neurons were photographed under phase contrast optics prior to anoxic exposure and were then relocated and photographed after varying intervals of anoxia. A1, Field just prior to exposure to anoxic environment showing clusters of neuronal cell bodies and interconnecting neurites. A2, After $8 \mathrm{hr}$, neuronal cell bodies are hard to distinguish, although process outlines are partially preserved. $A 3$, After 1 day, cells have been totally replaced by debris, although it is still possible to see process outlines. B1, Field of neurons treated with $10^{-2} \mathrm{M}$ DGG prior to anoxia. B2, After $8 \mathrm{hr}$, most of the cell bodies remain intact, and processes are little changed. B3, After 1 day, there is little additional change, and neurons appear, at most, slightly altered. Scale: $50 \mu \mathrm{m}$.

with cis-2,3-piperidine dicarboxylate, another excitatory amino acid antagonist (Davies et al., 1981), were unsuccessful because that agent was directly toxic when applied for long periods.

Intracellular recording provided additional evidence that neurons in the DGG-treated cultures were alive and healthy. Media and DGG were removed from one culture and replaced with standard recording HBSS after $18 \mathrm{hr}$ of anoxia. The average resting membrane potential of 12 consecutively impaled neurons was $65.3 \pm 6.2 \mathrm{mV}$. This compares favorably with an average resting potential of $62.5 \pm 6.8 \mathrm{mV}$ found in a series of 251 cultured hippocampal neurons previously studied in this laboratory and also with literature values for pyramidal cells in the 
hippocampal slice (Schwartzkroin, 1977). Action potential amplitude in eight consecutive cells was $70 \pm 6.8$ $\mathrm{mV}$, which is also in the range seen in the slice preparation. It was impossible to perform this experiment in anoxic control cultures, because there were no identifiable neuronal cell bodies after more than a few hours of anoxia.

After the protective effect of DGG was established, the neurotoxicity of GLU and ASP was investigated. When culture medium was replaced with MEM, to which $10^{-4}$ M GLU or $10^{-4} \mathrm{M}$ ASP had been added $(n=3$ in both cases), virtually all neurons, but not the glia, were destroyed within $1 \mathrm{hr}$ (Fig. $2, A$ and $C$ ). There was no apparent selectivity in that neuronal death was obvious in the entire culture. GLU and ASP were equally effective in killing neurons when TTX, $1 \mu \mathrm{g} / \mathrm{ml}$, and magnesium, $10 \mathrm{mM}$, were added to the bath (photographs not shown). However, when $10^{-2}$ M DGG was added to MEM, along with either GLU or ASP ( $n=3$ in both cases), no changes were visible in the neurons, viewed under phase contrast optics (Fig. 2, $B$ and $D$ ). It was quite clear that DGG was capable of dramatically blocking the neurotoxicity of exogenous GLU and ASP.

Intracellular recording provided additional information about the physiological and pharmacological properties of DGG. All cells studied had resting membrane potentials of $\geq 49 \mathrm{mV}$.

Direct application of $10^{-2}$ M DGG by microperfusion had no effect on the resting membrane potential or input impedance of five consecutive neurons (Fig. $3, A 1$ and A2). Both brief $(100 \mathrm{msec})$ and prolonged $(1 \mathrm{sec})$ applications were tried. DGG did have a marked effect on the depolarization produced by microperfusion of GLU and ASP (Fig. 3, B1 and B2). The simultaneous application of GLU and DGG, $10^{-4} \mathrm{M}$ and $10^{-2} \mathrm{M}$, respectively, resulted in $91.6 \pm 11.8 \%$ reduction in the depolarization produced by GLU alone $(n=5)$. Three cells showed no depolarization in the presence of DGG. When DGG and ASP were applied together to five consecutive cells, no depolarization was seen in four, and in the fifth cell there was a $90 \%$ reduction in the response.

Finally, the effect of DGG, $10^{-2} \mathrm{M}$, on excitatory transmission was examined. Pairs of neurons in the same microscope field were impaled and separately stimulated via the bridge circuit to determine whether they were synaptically coupled. When pairs were found, such that an action potential in one cell elicited an EPSP of short, fixed latency in the second, DGG was applied for approximately $20 \mathrm{sec}$, and the EPSP was monitored. In seven consecutive pairs of connected cells, the EPSP decreased by $86.1 \pm 18.9 \%$ after DGG; in four of these pairs the EPSP disappeared completely (Figs. 3, C1 to $C 3$, and $4, A 1$ to $A 3$ ). These effects were reversible in all cases. Those cells which were spontaneously active showed a marked diminution in the size of EPSPs and the frequency of action potentials. While IPSPs were not systematically studied, no DGG effect was seen on the two IPSPs encountered (Fig. 4).

\section{Discussion}

The experiments described above show that DGG, a postsynaptic blocker of excitatory amino acids, markedly decreases the size of EPSPs in coupled neurons and prevents the death of anoxic hippocampal neurons in vitro. These results provide strong support for the hypothesis that synaptically released neurotransmitter, most likely GLU or ASP, is responsible for the death of anoxic neurons, at least in this in vitro system. This conclusion is further supported by the prior observation that blockade of transmitter release is also effective in preventing anoxic neuronal death (Rothman, 1983).

It is beyond the scope of this paper to summarize all of the pertinent data relating to GLU and ASP as potential transmitters, especially since this subject has been recently reviewed (Roberts et al., 1981). There is abundant evidence that hippocampal pyramidal cells in vivo release GLU and/or ASP as their excitatory transmitters (Strom-Mathisen and Opsahl, 1978; Malthe-Sorensson et al., 1979, 1980). In addition, EPSPs and GLU and ASP depolarizations in cultured hippocampal neurons, many of which are derived from pyramidal neurons (Banker and Cowan, 1977; Rothman and Cowan, 1981), have been blocked by DGG and cis-2,3-piperidine dicarboxylate, another antagonist of excitatory amino acids (Rothman, 1982). Further evidence supporting a transmitter role for GLU or ASP in these cultures is that they are the only putative excitatory transmitters which invariably depolarize neurons in this system. No consistent depolarizations are produced by acetylcholine, norepinephrine, serotonin, somatostatin, synthetic enkephalins, or adenosine (Rothman, 1980, and unpublished results). Thus, there is good reason to believe that GLU, ASP, or a very closely related chemical is the excitatory neurotransmitter utilized by cultured hippocampal neurons.

However, there are three other questions which need to be considered: (1) Does DGG act other than as an excitatory amino acid antagonist? (2) Are excitalory amino acids directly toxic to neurons, or are their effects mediated by synaptic activity? (3) Why are GLU and ASP not damaging to the central nervous system under normoxic conditions?

The experiments described above found no evidence that DGG, even at $10^{-2} \mathrm{M}$, had any direct, postsynaptic effects on these cultured neurons; no changes were observed in resting potential or input impedance. Although not examined systematically, action potentials appeared unchanged during DGG microperfusion. Amino acidinduced depolarizations and EPSPs were both comparably antagonized by DGG (approximately $90 \%$ reduction for both), arguing that DGG functions exclusively as a blocker of the postsynaptic effects of excitatory amino acids. That two IPSPs in synaptically connected neurons were unaffected by DGG argues against DGG having a nonspecific effect on synaptic transmission. More compelling evidence for an exclusive, postsynaptic site of action for DGG comes from recent experiments in the hippocampal slice, which showed that DGG was capable of antagonizing the perforant path-granule cell EPSP and also the direct postsynaptic effects of GLU and ASP (Crunelli et al., 1983). A quantal analysis showed that the EPSP reduction was caused by a decrease in quantal size rather than quantal content, a result inconsistent with a presynaptic action of DGG. 

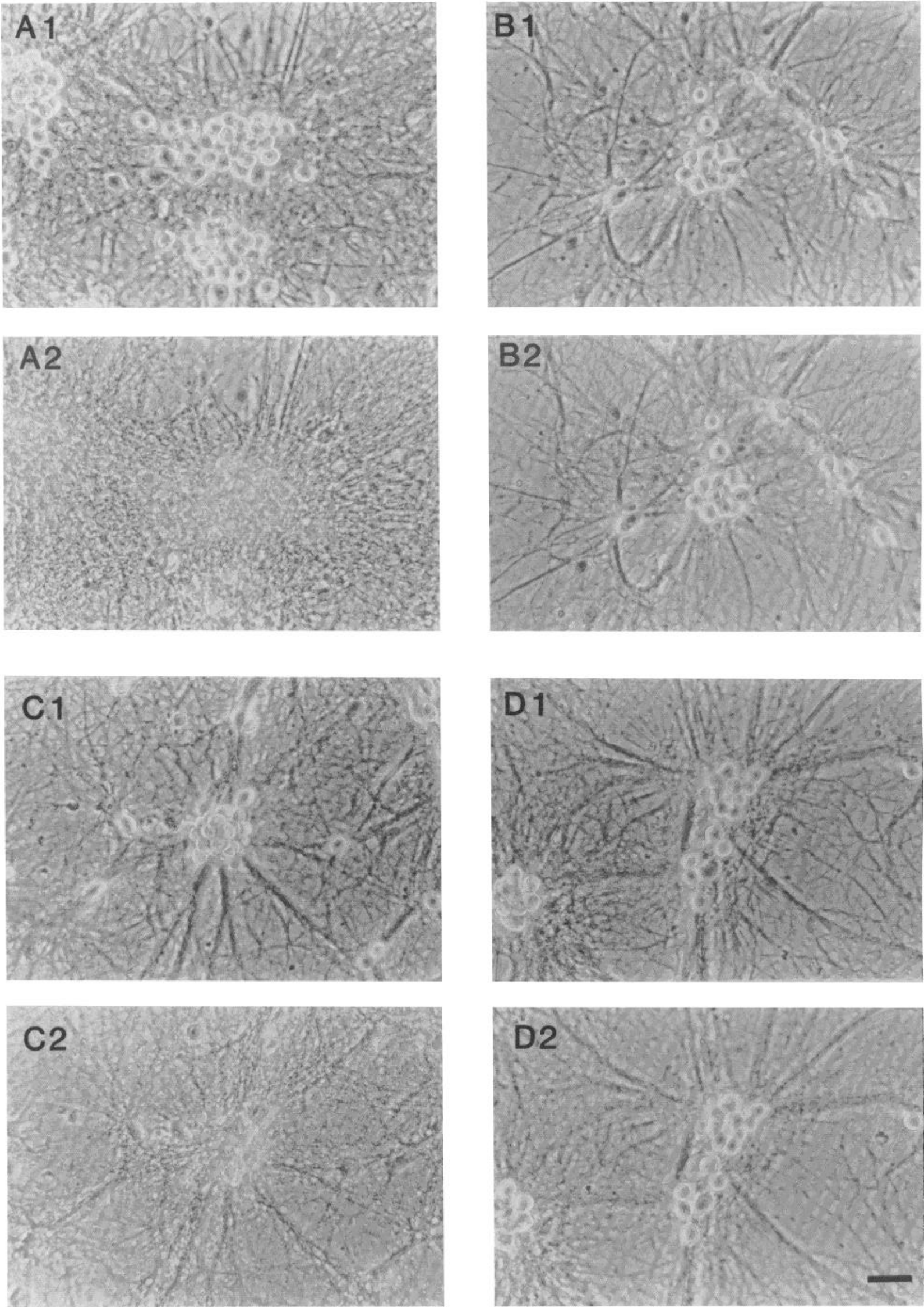

Figure 2 

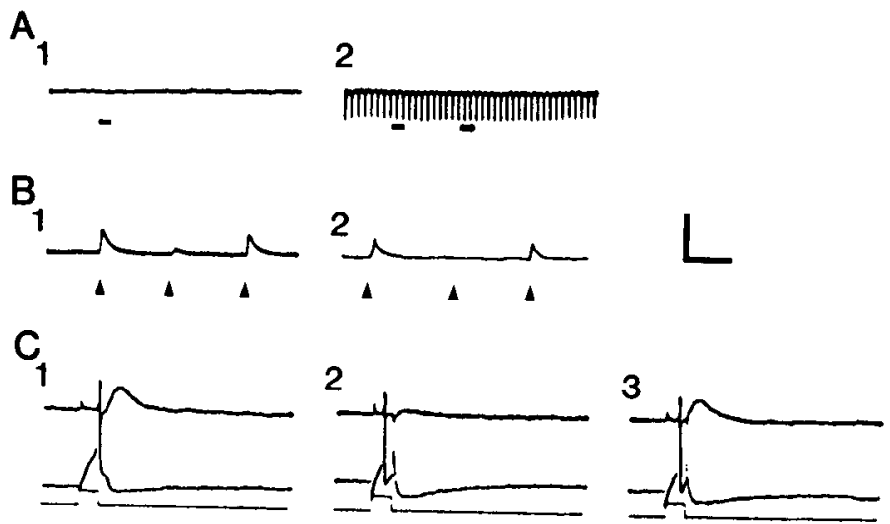

Figure 3. Physiological effects of DGG. 11, A 100-msec application (line) of $10^{-2} \mathrm{M} D G G$ did not alter the resting potential of a cultured hippocampal neuron. A2, Longer applications of DGG (lines), lasting $1 \mathrm{sec}$, failed to change the resting potential or input impedance of the same neuron. Impedance was measured by passing $60-\mathrm{msec}, 0.5-\mathrm{nA}$ current pulses through the bridge. B1, Application of $10^{-4} \mathrm{M}$ GLU alone for $100 \mathrm{msec}$ at the first and third time markers produced an immediate depolarization. Simultaneous application of GLU and $10^{-2} \mathrm{M}$ DGG at the second time marker produced almost no response. $B 2$, This sequence shows that $10^{-4} \mathrm{M}$ ASP produced an immediate depolarization also, when applied at the first and third arrows for $200 \mathrm{msec}$. DGG, $10^{-2} \mathrm{M}$, completely blocked this effect, when both were simultaneously applied at the second time marker. $C$, This sequence shows the effect of $10^{-2} \mathrm{M}$ DGG on the FPSP produced by intracellular stimulation of the presynaptic neuron. In $C 1$ to $C 3$, the upper trace is postsynaptic voltage, the middle trace is presynaptic voltage, and the bottom trace is current injected into the presynaptic neuron. C1, EPSP of approximately $10 \mathrm{mV}$ followed presynaptic action potential. $C 2$, Immediately after a 20 -sec application of DGG close to both neurons, the EPSP was almost completely blocked. C3, After approximately $20 \mathrm{sec}$, the EPSP returned. Membrane potentials: $A 1$ and $A 2,-73 \mathrm{mV} ; B 1,-66$ $\mathrm{mV} ; B 2,-68 \mathrm{mV} ; C$, presynaptic $-70 \mathrm{mV}$, postsynaptic -74 $\mathrm{mV}$. Voltage calibration is $40 \mathrm{mV}$ for all traces except postsynaptic trace in $C$, where it is $20 \mathrm{mV}$. Current calibration is $5 \mathrm{nA}$ in $C$. Time base is $400 \mathrm{msec}$ for $A 1 ; 4 \mathrm{sec}$ for $A 2, B 1$, and $B 2$; 20 msec for $C$.

It might be expected that synaptically released GLU or ASP would depolarize neurons and increase the frequency of action potentials and synaptic potentials. This could lead to neuronal death in an anoxic atmosphere if neurons were unable to keep up with the metabolic demands imposed by increased electrical activity. The burden of maintaining ionic gradients, as well as anabolic functions, might overwhelm a cell that had only anaerobic metabolism available. The observation that GLU and ASP are equally toxic in the presence of TTX and high magnesium, which should prevent sodium action potentials and synaptic potentials, suggests that this is
A

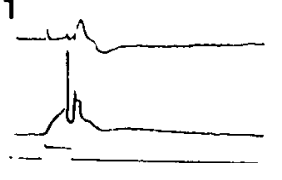

2

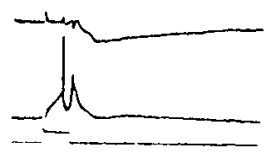

3

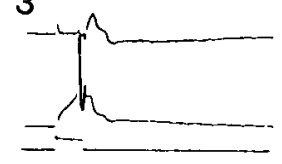

$\mathrm{B}_{1}$

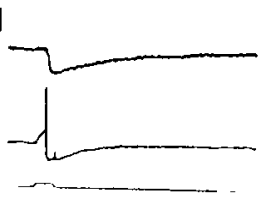

2
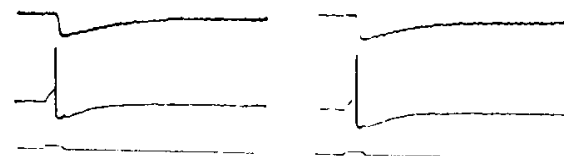

Figure 4. Synaptic physiology of DGG. These sequences show control synaptic potentials in pairs of connected neurons (1), the effect of a 20-sec application of DGG (2), and then the return to control levels after $20 \mathrm{sec}(3)$. The trace arrangement is identical to Figure $3 C$. A, Presynaptic action potential generated a short latency, presumably monosynaptic EPSP, and a longer latency polysynaptic IPSP. The EPSP almost completely disappeared after DGG, but the IPSP was unaffected. The excitatory input to the inhibitory neuron, which then produced the IPSP, was probably distant from the site of DGG microperfusion. $B$, In another pair of neurons in which a short latency IPSP was always produced by stimulation of the presynaptic neuron, no change was seen in the IPSP after DGG. Membrane potentials: $A$, presynaptic $-74 \mathrm{mV}$, postsynaptic $-68 \mathrm{mV} ; B$, presynaptic $-49 \mathrm{mV}$, postsynaptic $-69 \mathrm{mV}$. Calibration is $40 \mathrm{mV}$ for presynaptic traces, $20 \mathrm{mV}$ for postsynaptic traces, and $5 \mathrm{nA}$ for current traces in both $A$ and $B$. The time base is $20 \mathrm{msec}$ for $A$ and $40 \mathrm{msec}$ for $B$.

not the case. This finding is more consistent with a direct, toxic effect of excitatory transmitter that is independent of active membrane properties.

Excessive calcium influx has recently been implicated as the cause of hepatocyte death after toxin exposure (Schanne et al., 1979) and agonist-induced muscle injury (Leonard and Salpeter, 1979). GLU and other excitatory amino acids activate calcium entry into neurons (Berdichevsky et al., 1983), so abnormal calcium influx is an appealing explanation for their neurotoxicity. This may be the mechanism by which GLU induces degeneration of locust muscle (Donaldson et al., 1983). However, GLU and related excitatory amino acids are neurotoxic in medium which contains no added calcium (S. Rothman, manuscript in preparation). This observation fails to support the hypothesis that calcium entry is responsible for the neurotoxicity of excitatory amino acids (Berdichevsky et al., 1983).

A recent observation, that cultured hippocampal neurons are protected from GLU if an impermeable anion is substituted for external chloride, suggests a more plausible mechanism of cell death (S. Rothman, manuscript

Figure 2. Effects of $10^{-4} \mathrm{M}$ GLU and ASP. Representative fields were photographed prior to addition of GLU or ASP and were then relocated and photographed after $1 \mathrm{hr}$. $A$, This sequence shows the same field before $(A 1)$ and then $1 \mathrm{hr}$ after $(A 2) \mathrm{GLU}$ exposure. The neurons have all been replaced by debris over this period. $B$, This sequence shows a field in a culture treated with $10^{-2}$ M DGG prior to the addition of GLU. The culture appears identical before $(B 1)$, and 1 hr after $(B 2)$ GLU exposure. $C$, This sequence shows the appearance of a typical field prior to (C1) and $1 \mathrm{hr}$ after $(C 2)$ addition of ASP. D, This sequence demonstrates the lack of ASP toxicity in a culture pretreated with DGG. The initial appearance of the neurons $(D 1)$ has not changed after 1 hr (D2). Scale: $50 \mu \mathrm{m}$. 
in preparation). Chloride influx, triggered by sustained GLU depolarization, produces additional inward cation and water fluxes, which eventually result in cell lysis. Thus, secondary ion fluxes, and not depolarization per $s e$, may be responsible for neuronal death associated with GLU.

If natural neurotransmitters are neurotoxic, how does the central nervous system survive? Under most circumstances, a very efficient uptake system in nerve terminals (Storm-Mathisen and Iverson, 1979) and glia (Hertz, 1979) rapidly clears the extracellular space of synaptically released GLU and ASP, and the concentration of these transmitters never reaches toxic levels. The results described in this paper could be explained by an uptake system which fails to operate under anoxic conditions, allowing GLU and ASP to accumulate. Although there is no direct experimental evidence supporting this hypothesis, neurotransmitter uptake is highly energy dependent, so vulnerability to anoxia would not be unexpected (Hertz, 1979). The fresh N2 culture medium contains $50 \mu \mathrm{M}$ GLU and ASP, respectively, which the neurons tolerate. After 2 weeks in vitro, the time of most of these experiments, the GLU concentration drops to $30 \mu \mathrm{M}$ (I. Karl, personal communication). The threshold for GLU and ASP toxicity is approximately $100 \mu \mathrm{M}$, so there is a narrow range of tolerable excitatory amino acid concentrations, which could be exceeded if anoxia interfered with uptake.

Alternately, anoxia may increase the sensitivity of neurons to the toxic effects of GLU and ASP. This is an unlikely explanation for two reasons. First, previous experiments have shown that neurons survive in an anoxic atmosphere when synaptic activity is blocked (Rothman, 1983). In this situtation the neurons tolerated the GLU and ASP present in the full medium. As mentioned above, the GLU concentration would be $30 \mu \mathrm{M}$ in these cultures. Second, $100 \mu \mathrm{M}$ GLU or ASP rapidly kills neurons under normoxic conditions. Therefore, decreased oxygen tension is not a prerequisite for excitatory amino acid neurotoxicity.

There are at least two other situations in the mammalian central nervous system where excitatory amino acids may mediate neuronal death: (1) Repetitive focal motor seizures in rats produce cortical and thalamic neuronal degeneration resembling that caused by GLU and ASP (Collins and Olney, 1982). (2) Decreased systemic GLU catabolism may result in elevated nervous system GLU concentrations and neuronal degeneration in patients with olivopontocerebellar atrophy (Plaitakis et al., 1982). There is some precedent, then, for suggesting that excitatory amino acids are responsible for neuronal damage if their concentrations are elevated due to impaired uptake, excessive release, or slow catabolism.

At least three lines of evidence suggest that the results obtained in vitro will be shown to have wide validity. First, dentate granule cells in the hippocampal slice tolerate prolonged anoxia when bathed in extracellular buffer containing a high concentration of magnesium and no added calcium, which suppresses synaptic transmission (Kass and Lipton, 1982). Second, in a number of animal models of occlusive cerebrovascular disease (Michenfelder et al., 1976), early treatment with barbi- turates, which decreases synaptic activity (Macdonald and Barker, 1978), reduced the extent of brain infarction. Third, the neurons in the brain most sensitive to ischemic and hypoxic damage (CAI pyramidal cells, Purkinje cells, striatal neurons, and cortical neurons of layer III) are contacted by synaptic terminals containing excitatory amino acids, implying a role for these transmitters in triggering neuronal destruction (Jorgensen and Diemer, 1982). Therefore, there is reason to believe that synaptic activity, and specifically released GLU and ASP, mediate hypoxic neuronal injury in vivo.

These experiments may have far-reaching therapeutic implications for clinical neurology. It may prove possible to protect the human brain from hypoxic and/or ischemic insults by selectively preventing release of specific excitatory amino acids or blocking their postsynaptic actions. It is unlikcly that DGG will prove useful in this role because millimolar concentrations were required to demonstrate an effect in culture. However, it is reasonable to expect that more potent blockers of excitatory amino acids will be developed, and these should have widespread applications.

\section{References}

Banker, G. A. and W. Cowan (1977) Rat hippocampal neurons in dispersed cell culture. Brain Res. 176: 397-425.

Berdichevsky, E., N. Riveros, S. Sanchez-Armass, and F. Orrego (1983) Kainate, $N$-methylaspartate and other excitatory amino acids increase calcium influx into rat brain cortex cells in vitro. Neurosci. Lett. 36: 75-80.

Bottenstein, J., and G. Sato (1979) Growth of a rat neuroblastoma cell line in serum-free supplemented medium. Proc. Natl. Acad. Sci. U. S. A. 76: 514-517.

Choi, D. W., and G. Fischbach (1981) GABA conductance of chick spinal cord and dorsal root ganglion neurons in cell culture. J. Neurophysiol. 45: 605-620.

Collins, R. C., and J. Olney (1982) Focal cortical seizures cause distant thalamic lesions. Science 218: 177-179.

Crunelli, V., S. Forda, and J. Kelly (1983) Blockade of amino acid-induced depolarizations and inhibition of excitatory post-synaptic potentials in rat dentate gyrus. J. Physiol. (Lond.) 341: 627-640.

Davies, J., R. Evans, A. Francis, A. Jones, and J. Watkins (1981) Antagonism of excitatory amino acid-induced and synaptic excitation of spinal neurones by cis-2,3-piperdine dicarboxylate. J. Neurochem. 36: 1305-1307.

Donaldson, P. L., I. Duce, and P. Usherwood (1983) Calcium accumulation precedes the degenerative effects of L-glutamate on locust muscle fibers. Brain Res. 274: 261-265.

Francis, A., A. Jones, and J. Watkins (1980) Dipeptide antagonists of amino acid-induced and synaptic excitation in the frog spinal cord. J. Neurochem. 35: 1458-1460.

Hertz, L. (1979) Functional interactions between neurons and astrocytes. I. Turnover and metabolism of putative amino acid transmitters. Prog. Neurobiol. 13: 277-323.

Jorgensen, M. B., and N. Diemer (1982) Selective neuron loss after cerebral ischemia in the rat: Possible role of transmitter glutamate. Acta Neurol. Scand. 66: 536-546.

Kass, I., and P. Lipton (1982) Mechanisms involved in irreversible anoxic damage to the in vitro rat hippocampal slice. J. Physiol. (Lond.) 332: 459-479.

Leonard, J. P., and M. Salpeter (1979) Agonist-induced myopathy at the neuromuscular junction is mediated by calcium. J. Cell Biol. 82: 811-819.

Macdonald, R. L., and J. Barker (1978) Different actions of 
anticonvulsant and anesthetic barbiturates revealed by use of cultured mammalian neurons. Science 200: 775-777.

Malthe-Sorenssen, D., K. Skrede, and F. Fonnum (1979) Calcium-dependent release of $\mathrm{D}-\left[{ }^{3} \mathrm{H}\right]$ aspartate evoked by selective electrical stimulation of excitatory afferent fibres to hippocampal pyramidal cells in vitro. Neuroscience 4 : 12551263.

Malthe-Sorenssen, D., K. Skrede, and F. Fonnum (1980) Release of $\mathrm{D}-\left[{ }^{3} \mathrm{H}\right]$ aspartate from the dorsolateral septum after electrical stimulation of the fimbria in vitro. Neuroscience 5 . 127-133.

Michenfelder, J. D., J. Milde, and T. Sundt (1976) Cerebral protection by barbiturate anesthesia. Arch. Neurol. 33: 345350.

Olney, J. W. (1978) Neurotoxicity of excitatory amino acids. In Kainic Acid as a Tool in Neurobiology, E. McGeer, J. Olney, and P. McGeer, eds., pp. 95-121, Raven Press, New York.

Plaitakis, A., S. Berl, and M. Yahr (1982) Abnormal glutamate metabolism in an adult-onset degenerative neurological disorders. Science 216: 193-196.

Raichle, M. E. (1983) The pathophysiology of brain ischemia. Ann. Neurol. 13: 2-10.

Ransom, B. R., E. Neale, M. Henkart, P. Bullock, and P. Nelson (1977) Mouse spinal cord in cell culture. I. Morphology and intrinsic neuronal electrophysiologic properties. J. Neurophysiol. 40: 1132-1150.

Roberts, P. J., J. Storm-Mathisen, and G. Johnston (1981) Glutamate. Transmitter in the Central Nervous System, Wiley-Interscience, New York.
Rothman, S. (1980) Development and transmitter sensitivity of dispersed fetal hippocampal neurons in culture. Soc. Neurosci. Abstr. 6: 290.

Rothman, S. (1982) Synaptic physiology of dissociated hippocampal cultures. Soc. Neurosci. Abstr. 8: 800.

Rothman, S. M. (1983) Synaptic activity mediates death of hypoxic neurons. Science 220:536-537.

Rothman, S. and W. Cowan (1981) A scanning electron microscope study of the in vitro development of dissociated hippocampal cells. J. Comp. Neurol. 195: 141-155.

Schanne, F. A., A. Kane, F. Young, and J. Farber (1979) Calcium dependence of toxic cell death: A final common pathway. Science 206: 699-700.

Schwartzkroin, P. A. (1977) Further characteristics of hippocampal CAI cells in vitro. Brain Res. 128: 53-68.

Seifert, W., B. Ranscht, H. Fink, H. Muller, and E. Furster (1981) The developing hippocampus in cell culture. Soc. Neuroci. Abstr. 7: 768.

Seisjo, B. K. (1981) Cell damage in the brain: A speculative synthesis. Cereb. J. Blood Flow Metab. 1: 155-185.

Storm-Mathisen, J., and M. Opsahl (1978) Aspartate and/or glutamate may be transmitters in hippocampal efferents to septum and hypothalamus. Neurosci. Lett. $9: 65-70$.

Storm-Mathisen, J., and L. Iversen (1979) Uptake of $\left[{ }^{3} \mathrm{H}\right]$ glutamic acid in excitatory nerve endings: Light and electron microscopic observations in the hippocampal formation of the rat. Néuroscience 4: 1237-1253. 